\title{
Dispersed Electrical-Relaxation Response: Discrimination Between Conductive and Dielectric Relaxation Processes
}

\author{
J. Ross Macdonald* \\ Department of Physics and Astronomy \\ University of North Carolina \\ Chapel Hill, NC 27599-3255, USA
}

Received 9 February, 1998; received in revised form 24 June, 1998

\begin{abstract}
Relations and distinctions which are relevant to small-signal electrical-relaxation behavior are reviewed and applied to the important problem of identifying the physical processes leading to dispersed relaxation response. Complex-nonlinear-least-squares fitting of a response model to frequency-response data is found not to allow one to distinguish unambiguously in most cases between conductive-system response of Wagner-Voigt type, which may be characterized by a distribution of conductive-system relaxation times [DCRT], and dielectric- system response of Maxwell type, characterized by a distribution of dielectric-system relaxation times [DDRT]. In general, one must include a parallel conductivity element, $\sigma_{C P}$, as well as a high-frequencylimiting dielectric-system dielectric constant, in a conductive-system fitting model used to represent dielectric-system data with non-zero dc conductivity. Contrary to earlier predictions of Gross and Meixner, accurate numerical inversion of a set of exact frequency- response data to estimate the distribution with which it is associated shows that no discrete line necessarily appears in a DCRT associated with a truncated continuous DDRT. A discrete line can appear in general, however, when $\sigma_{C P} \neq 0$ and is unaccounted for in an inversion process. The novel result is established that a data set mathematically described in terms of a dielectric system with dc leakage and involving a Maxwell-circuit exponential distribution of relaxation times may be well represented within usual experimental error by a Wagner-Voigt conductive system involving a form of the important Kohlrausch-Williams-Watts response model.
\end{abstract}

\section{Introduction}

An important problem in analyzing the small-signal electrical frequency response of solids and liquids is to determine whether the response arises principally from mobile charges or from dielectric effects, such as rotating and/or induced dipoles. Although one sometimes knows enough about the material being analyzed to identify the type of electrical processes present in it, there are usually many sources of ambiguity that may make it difficult to distinguish between dispersive behavior arising from charges which can percolate through the entire material at low frequencies or from dispersion associated with dielectric processes, especially for data at only a single temperature. Given a set of isothermal electrical frequency-response data, the present work is concerned with the question of how best to analyze the data to identify the dominant dispersion process and so how to use macroscopic measurements to gain some mi- croscopic understanding of important physico-chemical processes present. A list of acronyms is included at the end of the present work.

For the electrical response area one defines four related immittance levels, which, expressed in terms of specific quantities, are (a) the complex resistivity level, $\rho(\omega)$; (b) the complex modulus level, $M(\omega) \equiv$ $i \omega \varepsilon_{V} \rho(\omega)$; (c) the complex dielectric constant level, $\varepsilon(\omega) \equiv[M(\omega)]^{-1}$; and (d) the complex conductivity level, $\sigma(\omega) \equiv i \omega \varepsilon_{V} \varepsilon(\omega)$ [1]. Here $\varepsilon_{V}$ is the permittivity of vacuum and $\varepsilon_{V} \varepsilon$ is the permittivity associated with a dielectric constant $\varepsilon$. It is important to note that while a given response model is usually most appropriately defined at a particular immittance level, its response can be expressed at any of the four levels. Similarly, data with small errors will yield closely similar fits to an appropriate model at any of the four levels, and exact synthetic data may be fitted exactly at any of the four levels by the model from which it was generated.

*E-mail: macd@email.unc.edu 
Consider first frequency-response data that are obtained from measurements on a pure dielectric material, one with no intrinsic dc conduction but with dispersed ac relaxation response associated with dipole rotation. Although one can never extend measurements to such low frequencies that the complete absence of any $\mathrm{dc}$ conduction can be absolutely established, in practice if no traces of such conduction appear in the data at the lowest practical frequencies, it will be an adequate approximation to ignore the possibility of $\mathrm{dc}$ conduction when analyzing the data. One would then conclude that impurity and surface-leakage conduction are negligible and that intrinsic conduction of charged entities involves so large a band gap that it too is negligibly small at measurement temperatures of interest. We are then dealing, for all practical purposes, with pure dielectric dispersion response, denoted herein by dielectric-system dispersion [DSD].

Much experimental data of DSD type do show, however, some apparent dc conduction effects in the available frequency range. If such conduction is not itself dispersed and does not exhibit the same type of temperature dependence as does the peak dielectric loss frequency, it is unlikely to arise from the same processes as the DSD part of the response. Such combined response may be defined as full DSD.

There is, however, another important possibility. Suppose that, alternatively, there is no DSD present, but only a high-frequency-limiting dielectric constant, $\varepsilon_{D \infty}$, frequency-independent over the measurement range. The material can still, nevertheless, involve $\mathrm{dc}$ conduction and show dispersion arising from the hindered motion of mobile, charged monopoles, such as ions. In this case, it is reasonable to expect that the dc conductivity is an intrinsic part of the full dispersive response, so that the dc conductivity and the peak loss frequency of the imaginary part of the complex resistivity show the same or nearly the same, possibly thermally activated, thermal response $[2,3]$. We may define such behavior, exclusive of $\varepsilon_{D \infty}$ as pure conductive-system dispersion [CSD], and that with the always present $\varepsilon_{D \infty}>1$ as full CSD.

Although a material involving only pure DSD will approach zero conductivity at limiting low frequencies $[2,3]$, interestingly, in this dielectric case a non-zero lowfrequency limiting resistivity is present and is consistent with zero limiting conductivity: i.e., no dc conduction [4]. In the most difficult discrimination situations, one might wish to distinguish between a dispersed conductive system with completely blocking electrodes and a dispersed dielectric system without dc leakage, or between a CSD situation without completely blocking electrodes and a pure dielectric system with dc leak- age resistance. In the present work, the second of these possibilities, one which often arises in practice, will be emphasized.

We shall deal both with the frequency response directly and with the distributions of relaxation/retardation times [DRT] associated with CSD and DSD behavior. Although the distinction between relaxation and retardation times is useful for mechanical systems [5-9], it is of lesser importance for dielectric systems [10], and here we shall often denote

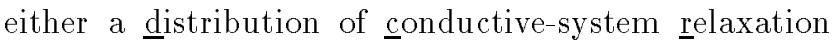
times [DCRT] or a distribution of dielectric-system relaxation imes [DDRT] by DRT. Then, just as it is important to define in the mechanical response area distributions of retardation times and distributions of relaxation times, and their inter-relationships, a subject pioneered by Bernhard Gross [5-9], it is appropriate in the electrical response area to consider both a DCRT and a DDRT and their interrelations.

But here a crucial distinction needs to be emphasized. Given a set of electrical frequency-response data, whether arising from CSD or from DSD, it is often possible to derive significant estimates of both a DCRT and a DDRT from the data. In such cases, further information is required to decide whether the data involve CSD or DSD. Nevertheless, estimating both a DCRT and a DDRT from a data set can often help one throw useful light on the discrimination problem mentioned above, especially since it is now possible to derive good estimates of the DRTs associated with a given temporal- or frequency-response data set. Although such estimation is generally an ill-posed inversion process, recent work shows how high-resolution results may be obtained from data which involve small or zero errors $[2,4,11]$.

In the past there has been considerable discussion about whether measured electrical response should be analyzed in terms of parallel processes (Maxwell systems) or series (serial) ones (termed Voigt or Wagner systems) [e.g., 1,12-15]. The present work addresses this question both implicitly and explicitly. At least formally, all such processes may be expressed in terms of DRTs, either discrete or continuous. Elemental Maxwell response, best defined at the dielectric constant level, involves $\mathbf{M}$ combinations of a resistor and capacitor in series (representing energy dissipation and storage, with each series combination defining a relaxation time) all in parallel [9]. When $\mathbf{M} \rightarrow \infty$ and differential elements are used, the resulting discrete DDRT becomes continuous. Such elemental Maxwell response leads to no dc conduction. In contrast, an elemental Wagner-Voigt system is the dual of the Maxwell one and involves $\mathbf{M}$ combinations of a resistor and capacitor 
in parallel, all in series, thus leading to a DCRT and to non-zero dc conduction. It is often called a Voigt circuit and is most sensibly defined at the complex resistivity level. One can also describe dispersed relaxation response in terms of $\mathbf{M}$ hierarchical RC elements (equivalently: ladder networks, transmission lines, or discrete continued-fraction expressions). These responses may involve a transmission line with either zero or non-zero dc conduction $[9,13]$.

Complete electrical response involving one of the above systems requires that a parallel leakage resistor be added if needed to those systems with no dc conductance and that a parallel capacitance, representing limiting high-frequency dielectric response always be present; the corresponding dielectric constant is $\varepsilon_{D \infty}$. Further, for a conducting system a non-zero high- frequency-limiting series resistor may be required [16], as well as a separate parallel conductance when a CSD model is used to fit leaky DRT response (see Section III-B below).

When one converts the full discrete-element Voigt model used for viscoelastic response [7] to an electrical equivalent circuit, one finds that it includes both a capacitor and a resistor in series with elemental Voigt response. Although it might be possible to fit CSD response with such a circuit if a resistor, representing $\mathrm{dc}$ conduction, were added in parallel with it, such a resistor would not be an integral part of the dispersed response model. Thus, the series capacitor is inappropriate for conducting systems with intrinsic non-zero $\mathrm{dc}$ conductivity and so must be removed, and the capacitance associated with $\varepsilon_{D \infty}$ must be added in parallel with the rest of the circuit. The resulting complete Voigt electrical response model, made up of discrete or continuous Voigt response elements with a possible resistor in series with them, all in parallel with the $\varepsilon_{D \infty}$ capacitance and a possibly-present independent paral- lel resistor, has not been previously considered. But there is substantial evidence that such a response model without the parallel resistor is appropriate for analyzing CSD data [2-4].

Although general dispersive response may involve both CSD and simultaneously present DSD $[17,18]$, we shall follow common usage here and consider only the presence of dispersion of one or the other type. The ac behavior of CSD systems has often in the past been represented by DDRT response instead of by probablymore-appropriate DCRT response [e.g., 12,14,15,19-22]. While it is relatively straightforward to identify thermally activated CSD response (even with blocking electrodes) and pure dielectric response (no dc conductivity), it is more difficult to discriminate between conductive and dielectric dispersion for other situations where only data at a single temperature are available.

Let us use the subscript $\mathrm{n}$, with $\mathrm{n}=\mathrm{D}$ to designate DSD response and/or fitting involving a DDRT-type model, and $n=C$ to designate CSD response and/or fitting involving a DCRT model. It proves convenient to further define two types of DCRT models (with $\mathrm{n}=$ $\mathrm{C} 0$ or $\mathrm{C} 1$, or just $\mathrm{n}=0$ or 1 in the following), whose responses we shall denote by CSD0 or CSD1. The important distinction between these two response types is discussed below.

\section{Some general response rela- tions}

Define $U_{n}$ as an unnormalized measured or model quantity of interest, such as a complex resistivity or complex dielectric constant. It is mathematically convenient to express the normalized form of $U_{n}, I_{n}$ in terms of a DRT, say $g_{n}(\tau)$. Let $x \equiv \tau / \tau_{\text {on }}$, where $\tau_{\text {on }}$ is a characteristic response time of the fitting model, and define $y \equiv \ln (x)$. We may now write $[1,2,4,23]$

$$
I_{n}(\omega) \equiv \frac{U_{n}(\omega)-U_{n}(\infty)}{U_{n}(0)-U_{n}(\infty)}=\int_{0}^{\infty} \frac{G_{n}(x) d x}{\left[1+i \omega \tau_{\mathrm{on}} x\right]}=\int_{-\infty}^{\infty} \frac{F_{n}(y) d y}{\left[1+i \omega \tau_{\mathrm{on}} \exp (y)\right]}
$$

where

$$
U_{n}(\omega)=U_{n}^{\prime}(\omega)+i \delta_{n} U_{n}^{\prime \prime}(\omega)
$$

and thus

$$
I_{n}(\omega)=I_{n}^{\prime}(\omega)+i \delta_{n} I_{n}^{\prime \prime}(\omega)
$$

It is important to emphasize that the choice $n=D$ specifies that the $U_{D}$ response of $\mathrm{Eq}$. (1) is that of the complex dielectric constant $\varepsilon(\omega)$ (or corresponding complex capacitance) and involves a distribution of dielectric-system relaxation times, a DDRT. On the 
other hand, the choices $n=0$ and $n=1$ specify response at the complex resistivity $\rho(\omega)$ (or impedance) level and thus involve, through $G_{0}$ and $G_{1}$, distributions of conductive-system relaxation times, DCRTs. We follow the usual sign conventions and set the quantities $\delta_{0}$ and $\delta_{1}$ in Eqs. (2) and (3) equal to 1 and $\delta_{D}$ equal to -1 .

Conservation of probability leads to the relations $G_{n}(x) \equiv \tau_{o n} g_{n}(\tau)$ and $F_{n}(y) \equiv x G_{n}(x)$. The $F_{n}$ form, which may be simply related to a distribution of activation energies [24], is particularly appropriate for numerical quadrature. Here the DRTs are normalized, so $I_{n}(0)=1$ and $I_{n}(\infty)=0$, as indicated above. Sometimes, one needs to deal with a cut-off distribution $[2,25]$. Then the limits of the integrals above may be changed from 0 to $\infty$ to $x_{\min }$ to $x_{\max }$, and from $-\infty$ to $\infty$ to $y_{\min }$ to $y_{\max }$.

The dimensionless moments of a general normalized DRT, $G_{n}(x)$, may be expressed as

$$
<x^{m}>_{n} \equiv \int_{0}^{\infty} x^{m} G_{n}(x) d x,
$$

with $m$ an integer. Then, for example, the average relaxation time for the distribution is $\langle\tau\rangle_{n}=\tau_{o n}<$ $x>_{n}$.

For CSD response, we set $U_{n}(\omega)=\rho_{C n}(\omega)$ with $n$ $=0$ or 1 , and define $\Delta \rho_{C n} \equiv \rho_{C n}(0)-\rho_{C n}(\infty)$. Herein $\rho_{C_{\infty}} \equiv \rho_{C n}(\infty)$ will be taken zero since it usually cannot be distinguished from zero when fitting CSD data $[2-4,23]$. Also define $\rho_{C o n} \equiv \rho_{C n}(0)$. Recent work $[2,23]$ has shown that for CSD response associated with a single DRT, e.g., $G_{n}(x)$, limiting dielectric constant contributions arising solely from CSD are

$$
\varepsilon_{C \infty n} \equiv \varepsilon_{\tau n} /<x^{-1}>_{n}
$$

and

$$
\varepsilon_{C o n} \equiv \varepsilon_{\tau n}<x>_{n}
$$

where in the general case

$$
\varepsilon_{\tau n} \equiv \tau_{o n} \Delta \rho_{C n} /\left[\varepsilon_{v}\left(\rho_{C o n}\right)^{2}\right] .
$$

For $n=1$ with $\rho_{C \infty}=0$, we obtain

$$
\varepsilon_{C \infty 1} \equiv \tau_{o 1} /\left(\varepsilon_{v} \rho_{C 01}<x^{-1}>_{1}\right) \text {. }
$$

The temporal relaxation function, $\phi_{n}(t)$, corresponding to the frequency response of Eq. (1) may be expressed as [2]

$$
\phi_{n}(t)=\int_{0}^{\infty} G_{n}(x) \exp \left(-t / \tau_{\text {on }} x\right) d x=\int_{-\infty}^{\infty} F_{n}(y) \exp \left\{-\left(t / \tau_{o n}\right) e^{-y}\right\} d y .
$$

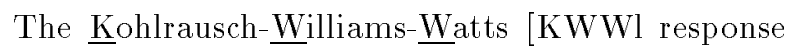
model [26], one which has been derived from various physical assumptions and found to represent a large body of data quite well $[2,3,13,23]$, involves fractional exponential time behavior for $\mathrm{n}=0$ or $\mathrm{D}$ (but not for $\mathrm{n}=1[25]$ ) and may be written as

$$
\phi_{n}(t)=\exp \left[-\left(t / \tau_{\text {on }}^{\beta_{n}}\right)\right], \quad 0<\beta_{n} \leq 1 .
$$

Although there is no closed-form expression available for KWW frequency response with arbitrary $\beta_{n}$, such response can be calculated numerically with very high accuracy and is available in fitting models incorporated in the free LEVM complex-nonlinear-least-squares computer program $[11,27]$ for all three values of $n$. These KWW models will be designated by KWWn.
For full CSD response ( $\mathrm{n}=0$ or 1 ) involving the Voigt circuit, and therefore associated with a DCRT, Eq. (1) may be rewritten at the complex resistivity level as

$$
\rho_{C n}(\omega)=\rho_{C \infty}+\Delta \rho_{C n} I_{n}(\omega),
$$

not including the effect of a necessary $\varepsilon_{D \infty}$ contribution. For DSD response $(n=D)$ involving the full Maxwell circuit, and therefore associated with a DDRT, we set $U_{D}(\omega)=\varepsilon_{D}(\omega)$, the complex dielectric constant, and obtain

$$
\varepsilon_{D}(\omega)=\varepsilon_{D \infty}+\Delta \varepsilon_{D} I_{D}(\omega)+\left(\sigma_{D 0} / i \omega \varepsilon_{V}\right),
$$

where $\Delta \varepsilon_{D}=\varepsilon_{D}(0)-\varepsilon_{D}(\infty), \varepsilon_{D \infty} \equiv \varepsilon_{D}(\infty)$, and $\sigma_{D \circ}$ 
is a possibly-present dc leakage conductivity, also designated as $\sigma_{0}$.

What is the difference between CSD0 and CSD1 behavior? Suppose that we are dealing with a particular form of the DRT $G_{0}(x)$, perhaps that associated with KWW0 response and often of the same form as $G_{D}(x)$. Then the corresponding $G_{1}(x)$ to use in Eq. (1) to obtain KWW1 response is defined as the normalized form of $x G_{o}(x)[2,23]$. It follows from Eq. (1) that

$$
G_{1}(x)=x G_{0}(x) /<x>_{o}
$$

and one finds that $\left\langle x^{-1}\right\rangle_{1}=1 /\langle x\rangle_{0}$, where the same parameter values must be used in these relations. Thus, one must use $\tau_{o 1}=\tau_{o 0}$ and $\beta_{1}=\beta_{0}$ in defining the KWW1 response which derives from KWW0. But note that CSD fitting of data with these separate models will yield different parameter estimates $[2,3,23]$.

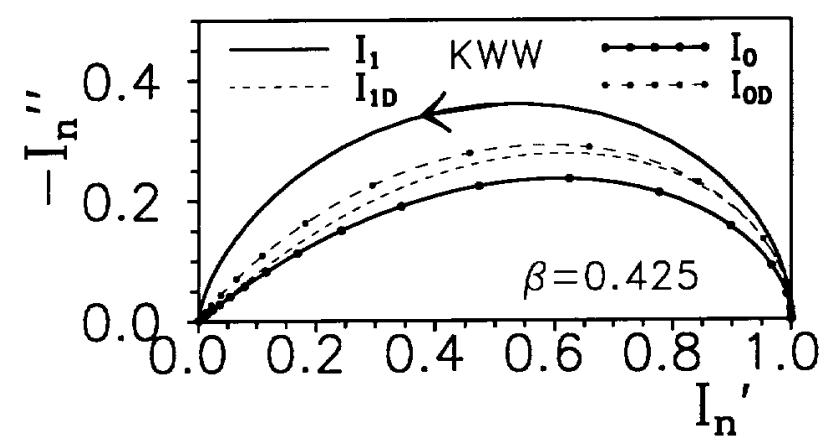

Figure 1. Complex plane plots of exact, normalized KWW frequency response. Curves $I_{1}$ and $I_{0}$ illustrate direct KWW1 and KWW0 complex resistivity response (solid lines), and $I_{1 D}$ and $I_{O D}$ show corresponding curves calculated by transforming the KWW data to the complex dielectric constant level and subtracting dc conductivity effects (dashed lines). The arrow shows the direction of increasing frequency.

Figure 1 presents complex plane plots of $I_{n}$ for $n=0$ and 1 , results which demonstrate some of the differences between exact KWW0 and the corresponding KWW1 model response. Because of the presence of the $x^{-}$ factor in $G_{1}(x)$, it yields response much closer to singlerelaxation-time Debye response than does KWW0 when $\beta_{1}=\beta_{0}$, the present choice. Now when complex- $\rho-$ level data (calculated as in Eq. (11) with $\rho_{C \infty}=0$ using the KWWn form of $G_{n}(x)$ ) are transformed to the complex $\varepsilon$ level, one can represent the results using Eq. (12). Then it is straightforward to use LEVM to remove the effects of $\varepsilon_{D \infty}$ and $\sigma_{D o}$ from the data in order to obtain the effective $I_{D}$ response associated with the original CSD data. To do so, we use the value of $\Delta \rho_{C n}=\rho_{C 0 n}$ employed in generating the data, calculate $\sigma_{D 0}=1 / \rho_{C 0 n}$ and set the $\varepsilon_{D \infty}$ in Eq. (12) equal to the value of $\varepsilon_{C \infty n}$ calculated using $\mathrm{Eq}$. (5), when the original $\rho(\omega)$ data were generated with $\varepsilon_{C \infty}=0$. It turns out that $\varepsilon_{C \infty 0}$ is zero for data involving a DCRT without high-frequency cutoff, but not $\varepsilon_{C \infty 1}$. Because the data were originally of CSD type, the resulting $I_{D}$ curves are denoted by $I_{1 D}$ and $I_{0 D}$. It is surprising that although the $I_{1}$ and $I_{0}$ curves are so different, those for $I_{1 D}$ and $I_{0 D}$ are quite similar.

\section{Direct data fitting}

\section{A. CSD-type data}

We shall start with exact synthetic CSD1 KWWl data generated using Eq. (11), with $I_{1}(\omega)$ set equal to the KWW1 model, and with parameter values the same as or close to those obtained with earlier KWW1 fitting of actual $321 \mathrm{~K} \mathrm{Na}_{2} \mathrm{O}-3 \mathrm{SiO}_{2}$ data with electrode effects eliminated $[4,23]$. The resulting CSD1 parameters are $\rho_{C 01}=1.45 \times 10^{9} \Omega-\mathrm{cm}, \tau_{01}=0.001 \mathrm{~s}$, and $\beta_{1}=0.425$. No $\epsilon_{\infty}$ value was initially included, and $\rho_{C \infty}$ was taken zero, in accordance with the results of earlier fits of the original data. The above values led to $\left\langle x^{-1}\right\rangle_{1} \simeq 0.3526,\left\langle x>_{1} \simeq 12.91, \epsilon_{C 01} \simeq 100.57\right.$, and $\epsilon_{C \infty 1} \simeq 22.09$, using Eqs. (4) through (8). These KWW1 values are summarized in row A of Table 1, where $\sigma_{C 0} \equiv\left(\rho_{C 01}\right)^{-1}$. In this table $S_{F}$ is the relative standard deviation of a fit, the standard deviation of the relative residuals. The synthetic data extended over the range $0.01 \leq \omega \leq 10^{10}$ radians $/ \mathrm{s}$ with 10 points per decade evenly spaced on a logarithmic scale. All the complex-nonlinear-least-squares fits of lines B, C, $\mathrm{E}$, and $\mathrm{F}$, and the fits discussed later, involved proportional weighting and used the LEVM V. 7.1 program $[27]$.

Next, the complex- $\rho$-level data of line A were transformed to the complex dielectric constant level, as denoted by the designation KWWID in row B. The fit results of lines $\mathrm{B}, \mathrm{C}, \mathrm{E}$, and $\mathrm{F}$ were obtained using $\mathrm{Eq}$. (12). Here, all the values shown in these lines are direct fit estimates with very small relative standard deviations (not shown). The $I_{D}(\omega)$ model function in this equation was the KWW expression for lines $B$ and $\mathrm{E}$, as specified by the $-\mathrm{K}$ part of KWWlD-K and was the exponential distribution of relaxation times [EDRT] model for lines $\mathrm{C}$ and $\mathrm{F}$, where $-\mathrm{E}$ appears. Although it 
has been conventional to use the symbol $\phi$ for the fractional exponent parameter of the EDRT model $[2,17]$, for simplicity it will be denoted by $\beta$ herein for easy comparison with KWW $\beta$ parameters.

Comparison of the results of lines $\mathrm{B}$ and $\mathrm{C}$ shows that the EDRT model provided a much better fit of the CSD data at the dielectric-constant level than did the KWW model. Further, except for the expected difference $[2,4,23]$ between the $\tau_{01}$ value of line $\mathrm{A}$ and the $\tau_{0 D}$ value of line $\mathrm{C}$, one sees that the line-C estimated parameter values are in excellent agreement with the exact ones of line A, and, specifically, that the $\sigma_{D 0}$ value of line $\mathrm{C}$ is an exceptionally close estimate of $1 / \rho_{C 01}$. Thus, although the $\epsilon_{D \infty}$ values of lines $\mathrm{B}$ and $\mathrm{C}$ are denoted by a subscript $n=D$ to indicate their estimation at the dielectric level by a complex-dielectric-constant response model, we see that they are, in fact, $\epsilon_{C \infty}$ estimates, necessary here because no actual DSD $\epsilon_{D \infty}$ values have been included, and we are dealing solely with CSD-type data, not DSD.

Usually, additional information will be required to distinguish between a true DSD data set and its transform fitted by a CSD approach, or, conversely, between a true CSD data set, and its transform fitted by a DSD approach. Nevertheless, we shall, for convenience, designate DSD data fitted by a CSD approach, as in Eq. (11), by CSD designations, and CSD data fitted by a DSD approach, as in Eq. (12), by the DSD designation.

Finally, synthetic data like that of line A were generated with a permittivity $\epsilon_{V} \epsilon_{S}$ in series with the rest of the response. Here the series dielectric constant $\epsilon_{S}=10$ is used to represent the effect of the capacitance of completely blocking electrodes. The value of 6.884 in line $\mathrm{D}$ is just the series combination of 22.09 and 10 , and $\left(\epsilon_{D \infty}+\Delta \epsilon_{D}\right)$ should equal 10 , as it does for line D. Here we see from lines $\mathrm{E}$ and $\mathrm{F}$ that although the $-\mathrm{K}$ model leads to a better fit than does the $-\mathrm{E}$ one, contrary to the results in lines $\mathrm{B}$ and $\mathrm{C}$, both fits are relatively poor and are much worse than that of line $\mathrm{C}$.

It is interesting that because of the presence of $\epsilon_{S}$ it is unnecessary to include a $\sigma_{D 0}$ dc conductivity in the fit models of lines $\mathrm{E}$ and $\mathrm{F}$, unlike the situation for lines B and C. When either $\epsilon_{S}$ or $\sigma_{D 0}$ is taken as a free parameter of the fit at the dielectric level, the initial values progressively change until neither one makes any contribution to the fit. Thus, while each can be accurately estimated from CSD1 fitting of the data, neither can be estimated by fitting at the dielectric level using Eq. (12)! For actual experimental data, if the presence of complete blocking were unrecognized, one might well conclude that dielectric-level data similar to that used to obtain the fit results of lines $\mathrm{E}$ and $\mathrm{F}$ were those of a pure dispersed dielectric material (true DSD response) without any dc resistive leakage. Here we know that it is not, and it is worth emphasizing that instead of the $3 \% S_{F}$ of the fit of line $\mathrm{E}$, transformation of the data to the complex $\rho$ or $\mathrm{M}$ level and its fitting with the appropriate CSD model, here the KWW1, as in line D of Table 1, yields an exact fit.

Although a zero value of $\epsilon_{D \infty}$ was used in generating the CSD data of rows $\mathrm{A}$ and $\mathrm{D}$, if a non-zero value had been included, as is always necessary for the analysis of experimental data, we would have found that the " $\epsilon_{D \infty}$ " estimate in lines $\mathrm{B}$ and $\mathrm{C}$ should have been designated as $\epsilon_{\infty}$ and would have included $\epsilon_{D \infty}$ since fitting of this kind can only yield estimates of $\epsilon_{\infty} \equiv \epsilon_{C \infty}+\epsilon_{D \infty}[2,3,23]$. Thus for actual CSD data, one can only estimate $\epsilon_{D \infty}$ directly from a CSD fit and then calculate $\epsilon_{C \infty}$ and $\epsilon_{C 0}$ from such fit results. In the absence of any DSD in the measured frequency range, one can take the actual, always present, $\epsilon_{D \infty}$ equal to $\epsilon_{D 0}$, and we must identify the apparent DSD and nonzero $\Delta \epsilon_{D}$ as arising solely from CSD. Finally, it is worth remarking that an EDRT response model was found most appropriate for fitting dispersion results for the conductive material $\mathrm{CaTiO}_{3}: 30 \% \mathrm{Al}^{3+}$ at low temperatures using DSD fitting at the dielectric level, as in row $\mathrm{C}$ of the present Table 1 [18]. The synthetic-data results of lines $\mathrm{B}$ and $\mathrm{C}$ perhaps suggest why a EDRT model was there found to yield a closer fit of the experimental data than the KWWD model.

\section{B. DSD-type data}

Let us now regard the best-fit EDRT results of row C of Table 1 as representing possible DSD response with non-negligible leakage resistance. For concreteness, the parameter estimates and EDRT response model of row $\mathrm{C}$ were used to generate a new exact data set. The data were then transformed to the complex resistivity level and fitted with Eq. (11) using several models, all with $\rho_{C \infty}=0$. The best-fit model found, was, not surprisingly, the KWW. Since leakage resistance can be of any value, in order to investigate its effects three different values of the dc conductivity, $\sigma_{0}=\sigma_{D 0}$, are used in column 7 for lines A, D, and G of Table 2. That for line $\mathrm{A}$ is the value shown in row $\mathrm{C}$ of Table 1 , designated as $\sigma_{C 0} \equiv 1 / \rho_{C 01}=\left(\Delta \rho_{C 1}\right)^{-1}$ hereafter, and those for lines $\mathrm{D}$ and $\mathrm{G}$ are ten times larger and smaller, respectively. 
Finally, all three of these sets of exact data implicitly rather than the 22.11 of row $\mathrm{C}$ of Table 1. involved $\epsilon_{D \infty}=10$, obtained by setting $\epsilon_{\infty}=32.11$

TABLE 1. DSD fitting results of exact CSD data calculated using Eq. (11) with the KWW1 model and the parameter values of rows A and D. These data sets were fit at the complex dielectric constant level using Eq. (12). Here $\epsilon_{S}$ is the dielectric constant of a series capacitance, and $-\mathrm{K}$ and $-\mathrm{E}$ designate the use in this equation of the KWWD model or the EDRT model, respectively. The presence of a non-zero value of $\epsilon_{S}$ changes the interpretation of the $\epsilon_{n \infty}$ and $\Delta \epsilon_{n}$ quantities, as discussed in the text. $\epsilon_{n \infty}$ equals either $\epsilon_{D \infty}$ or $\epsilon_{C \infty 1}$ as appropriate. $S_{F}$ is the relative standard deviation of a fit.

\begin{tabular}{|c|c|c|c|c|c|c|c|c|}
\hline Col/n & Model & $\epsilon_{\mathrm{S}}$ & $\epsilon_{\mathrm{n} \infty}$ & $\Delta \epsilon_{\mathrm{n}}$ & $10^{3} \tau_{\text {on }}(\mathrm{s})$ & $\beta_{\mathrm{n}}$ & $10^{10} \sigma_{\mathrm{n} 0}(\Omega-\mathrm{cm})^{-1}$ & $\mathrm{~S}_{\mathrm{F}}$ \\
\hline & & & & & & & & \\
\hline A/1 & KWW1 & ---- & 22.09 & 78.48 & 1 & 0.425 & 6.897 & 0 \\
\hline B/D & KWW1D-K & ---- & 22.18 & 80.51 & 1.37 & 0.438 & 6.911 & 0.0243 \\
\hline C/D & KWW1D-E & --- & 22.11 & 78.12 & 46.1 & 0.430 & 6.895 & 0.0073 \\
\hline & & & & & & & & \\
\hline D/1 & KWW1 & 10 & 6.884 & 3.116 & 1 & 0.425 & 6.897 & 0 \\
\hline E/D & KWW1D-K & 10 & 6.900 & 3.016 & 1.65 & 0.438 & $-\cdots$ & 0.0310 \\
\hline F/D & KWW1D-E & 10 & 6.864 & 3.267 & 1.30 & 0.401 & $-\cdots$ & 0.0574 \\
\hline
\end{tabular}

TABLE 2. CSD fitting results of exact DSD data calculated using Eq. (12) with the EDRT model, the parameter values of rows $\mathrm{A}, \mathrm{D}$, and $\mathrm{G}$, and $\Delta \epsilon_{D}=78.48$. These data sets were fit at the complex resistivity level using Eq. (11) with a KWW1 or KWW0 model, and a parallel conductivity, $\sigma_{C P}$. For the KWW0 fits, the $\epsilon_{D \infty}$-column values are actually those of $\epsilon_{\infty}=\epsilon_{C \infty n}+\epsilon_{D \infty}$. The dimensions of the $\sigma$ 's and of $1 / \Delta \rho_{C n}$ are $(\Omega-c m)^{-1}$. Here $\sigma_{0}$ is the total de conductivity.

\begin{tabular}{|c|c|c|c|c|c|c|c|c|c|}
\hline Col/n & Model & $\epsilon_{\mathrm{D} \infty}$ & $\epsilon_{\mathrm{C} \infty \mathrm{n}}$ & $10^{3} \tau_{\text {on }}(\mathrm{s})$ & $\boldsymbol{\beta}_{\mathrm{n}}$ & $10^{10} \sigma_{\mathbf{0}}$ & $\mathbf{1 0}^{10} \sigma_{\mathrm{CP}}$ & $1^{-9} \Delta \rho_{\mathrm{Cn}}$ & $\mathrm{S}_{\mathrm{F}}$ \\
\hline & & & & & & & & & \\
\hline $\mathrm{A} / \mathrm{D}$ & EDRT & 10 & ---- & 46.1 & 0.430 & 6.895 & ---- & ---- & 0 \\
\hline $\mathrm{B} / 1$ & $\mathrm{KWW1}$ & 9.92 & 22.16 & 1.004 & 0.425 & 6.895 & 0 & 1.450 & 0.0071 \\
\hline $\mathrm{C} / 0$ & $\mathrm{KWW0}$ & 32.14 & 0 & 9.01 & 0.568 & 6.908 & 2.125 & 2.095 & 0.0065 \\
\hline & & & & & & & & & \\
\hline D/D & EDRT & 10 & ---- & 46.1 & 0.430 & 68.95 & ---- & ---- & 0 \\
\hline E/1 & KWW1 & 9.67 & 22.44 & 0.999 & 0.422 & 69.02 & 61.94 & 1.413 & 0.0051 \\
\hline F/0 & KWW0 & 32.15 & 0 & 8.80 & 0.568 & 68.82 & 63.97 & 2.061 & 0.0057 \\
\hline & & & & & & & & & \\
\hline G/D & EDRT & 10 & ---- & 46.1 & 0.430 & 0.6895 & ---- & ---- & 0 \\
\hline H/1 & KWW1 & 9.75 & 22.31 & 1.033 & 0.425 & 0.6917 & -6.046 & 1.484 & 0.0093 \\
\hline I/0 & KWW0 & 32.16 & 0 & 9.04 & 0.567 & 0.6925 & -4.119 & 2.078 & 0.0104 \\
\hline
\end{tabular}

Comparison of the results shown in lines A of Table 1 and $\mathrm{B}$ of Table 2 shows that the EDRT model represents the original data at the dielectric level very well since the CSD fit results of line B are very close to the exact ones of Table 1, line A, completing the circle of transformations and fits. The numbers shown in the $\sigma_{C P}$ column of Table 2 are values of a paral- lel conductivity parameter included in the KWW1 fits. Non-zero values are required in order to obtain a good estimate of the total dc conductivity, $\sigma_{0}=\sigma_{C P}+\sigma_{C 0}$, when $\sigma_{C 0} \neq \sigma_{D 0}$. This is because the CSD constitutive equations, (5-8), hold for Eq. (11) fits, as indicated by the row-E fit of Table 2. As we see, the $\Delta \rho_{C 1}$ estimates in Table 2 are close to the original value of this quan- 
tity even when the total dc resistivity is very different. Incidentally, in those situations where a non-zero value of $\sigma_{C P}$ was required, taking $\rho_{C \infty}$ as a free fitting parameter instead of $\sigma_{C P}$ led to completely unsatisfactory fits.

Although the KWW0 fit of line $\mathrm{C}$ is slightly better than the KWW1 one of line $\mathrm{B}$, it requires a non-zero value of $\sigma_{C P}$, and, without it, the resulting $S_{F}$ had the much larger value of 0.024 . Further, since the original KWW1 data included no $\sigma_{C P}$, the non-zero KWW0 $\sigma_{C P}$ value is anomalous and helps one decide whether a KWW0 or KWW1 fit is more appropriate for CSD data, where one usually expects to find $\sigma_{C P}=0$. Comparison of the $\beta$ values shown in lines $B$ and $C, E$ and $\mathrm{F}$, and $\mathrm{H}$ and $\mathrm{I}$ indicate, nevertheless, that the relation $\beta_{0}+\beta_{1}=1$, proposed earlier [3,23], is closely obeyed.

The results in the table show that when the estimated value of $\sigma_{C P}$ is non-zero, the estimated parameter values in lines $\mathrm{E}$ and $\mathrm{H}$ are not as close to the ones used to generate the original KWW1 data as when it is zero, but the parameter values are still relatively close to the original exact-data values, and the CSD-fit values of $\sigma_{0}$ are good estimates of the proper dc conductivity. These results provide further useful discrimination information. For experimental CSD response, good fits of the data are nearly always found without the need to include a parallel $\sigma_{C P}$ fitting parameter, and negative estimates of this quantity are physically unlikely for a passive system. Thus, the presence of a significant nonzero CSD1 DCRT- model fitting estimate of $\sigma_{C P}$ is an excellent indication that one is not dealing with ordinary CSD response but probably with leaky dielectric dispersion.

The small but non-zero $S_{F}=0.0071$ value in row $\mathrm{B}$ of Table 2 is an indication that the KWW1 CSD1 fitting model, $I_{1}(\omega)$, is not quite entirely appropriate for $I_{D}(\omega)$ EDRT DSD data, and it is reasonable to assume that if an appropriate model were available, an exact fit would be found. For actual experimental data, where the errors present in the data generally lead to $S_{F}$ values greater than 0.0071 , the distinction is usually unimportant.

Figure 2 compares the $M^{\prime \prime}(\omega)$ curves for the fits of lines B, E, and $\mathrm{H}$ of Table 2 and for two smaller values of the dc conductivity. Particularly interesting is the double-peak curve present when $\sigma_{0}$ is smaller than $\sigma_{C 0}$. No trace of such behavior is apparent for any of the corresponding - $\rho "(\omega)$ curves. Because the line- $\mathrm{H}$ fit is the worst of the three, both the original data and the fit points are included, but only the data lines or points are presented for the other curves. Estimation of the DRTs associated with these cases can shed further light on their differences and is explored in the next section. When two peaks are present, the peak of the lowerfrequency one appears at lower and lower frequencies as $\sigma_{0}$ decreases, as illustrated by the curve with $r=0.01$. Finally, when there is no dc conductivity, this response disappears from the measurable frequency range.

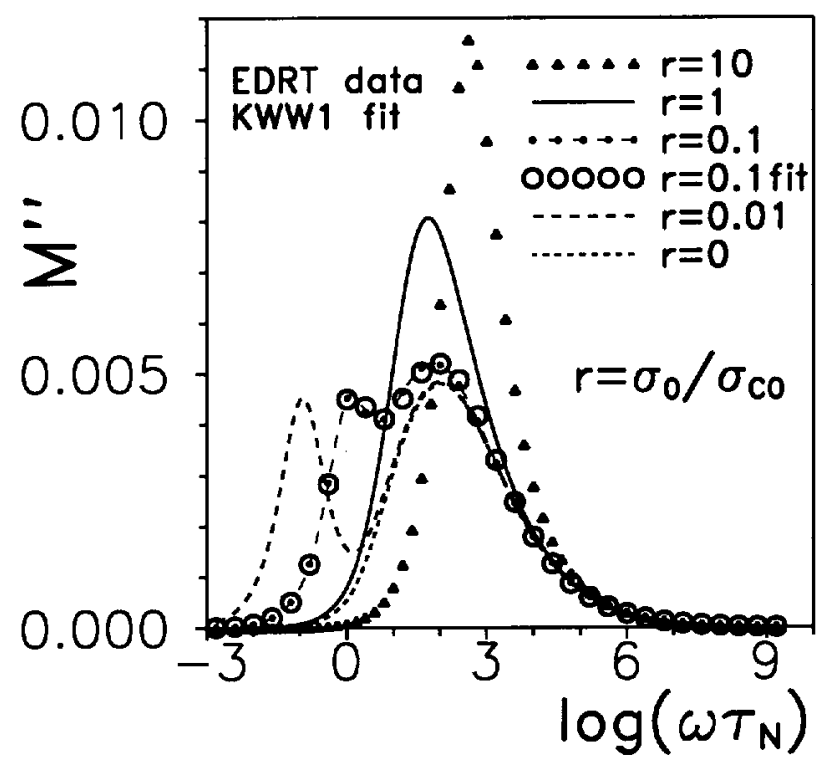

Figure 2. Comparison of the three $M "(\omega)$ data-line curves for rows A, D, and G of Table 2, and fit points for row $\mathrm{H}$ (open circles), and, as well, curves for two other smaller fixed values of $\sigma_{0}$. Here $r \equiv \sigma_{0} / \sigma_{0 A}$ where $\sigma_{0 A}=\sigma_{C 0}=$ $6.895 \times 10^{-10}(\Omega-\mathrm{cm})^{-1}$ is the dc conductivity included in the original exact EDRT dielectric data for row A of Table 2 , and the first three $\sigma_{0}$ values are those of rows D, A, and G of column 7 of Table 2. $\tau_{N}=1 \mathrm{~s}$ here and elsewhere.

\section{Data inversion and identifi- cation of dispersion types}

\section{A. Background}

Gross [5,7-9], Gross and Pelzer [6], and Kita [28] have been concerned with deriving analytic integral transforms connecting the DRTs associated with the $I_{1}(\omega)$ and $I_{D}(\omega)$ functions used to represent the same frequency-response data set. These transforms involve principal-value integration and, for DRTs only defined numerically, as is the case for the KWW model, they would be difficult to apply to yield accurate results, and, in fact, no transform results for such DRTs have been published. Therefore, here a different approach 
involving inversion is illustrated, one which yields DRT estimates which represent their associated frequency response with extremely high accuracy and do not require analytic expressions.

For the KWW model in the small- $x$ region, the $G_{0}(x)$ or $G_{D}(x)$ DRT is proportional to $x^{-\left(1-\beta_{n}\right)}$ and so diverges in the limit for $\beta_{0}$ or $\beta_{D}<1[2,29,30]$. Therefore, it is more instructive to plot $F_{D}$, which does not diverge in the limit, vs. $y$ or $\log (x)$, rather than to plot $G_{D}(x)$ vs. $\log (x)$. The above definitions and analysis show that for the KWW and other similar models $F_{D}$ and $G_{1}$ are proportional to $x G_{D}$ and $x G_{0}$ respectively, and do not diverge as $x \rightarrow 0$. For direct comparison of inversion results that estimate $G_{0}$ and $G_{1}$, or $G_{D}$ and $G_{1}$ from the same frequency-response data, it is most useful to compare the normalized distribution estimates of $F_{0}$ or $F_{D}$ and $G_{1}$. For accurate inversions, $F_{0}$ and $G_{1}$ should be identical. The LEVM program estimates $F_{1}$ directly for CSD1 analysis, but then outputs the corresponding $G_{1}$ while CSD0 or DSD inversion yields $F_{0}$ or $F_{D}$. For convenience, let $H(x)$ denote $F_{D}, F_{0}$, or $G_{1}$.

The high-resolution numerical inversion algorithm in the LEVM program yields a total of $M$ points of a continuous, discrete, or mixed distribution, where $M$ is presently limited to a maximum of 19 . Therefore, for such results, we replace $H(x)$ by $H_{i}$, with $1 \leq i \leq 19$. LEVM inversion yields estimates of the $M$ point pairs, $\left\{H_{i}, x_{i}\right\}$, where both quantities are free variables of the fit [11], not the case for other inversion techniques. Discrete and continuous-distribution points may therefore be unambiguously identified by comparing results for two different values of $M$ [11]. For continuous distributions, all $x_{i}$ estimates will be different for the two inversions, but the $x_{i}$ values of any discrete points present will remain the same. In the LEVM inversion algorithm, which uses numerical quadrature, it has not been found possible to account for end-point effects accurately for arbitrary distributions. Therefore, those $H_{i}$ estimates with the smallest, and sometimes largest $x_{i}$ values, and, to a lesser degree, their immediate nearest neighbors, will be less accurate than the remaining estimated points of the inversion $[4,11,30]$.

In the preceding section, we have shown by fitting of accurate numerical data that there is a very close connection between an EDRT representing a distribution of "dielectric" relaxation times and the corresponding KWW1 DRT representing a distribution of "resistivity" relaxation times: data generated by one of these response functions may be closely fit when the other is used instead, as demonstrated by the results presented in Tables 1 and 2. This connection has been further investigated by inversion, and some of the results obtained are presented in Figs. 3 through 5.

\section{B. DRT inversion estimates: Fig. 3 re- sults}

To obtain the DRT results shown in Fig. 3, CSD1type KWW1 frequency response data were produced using the parameters of row A of Table 1, except that $\epsilon_{D \infty}$ was usually taken as 10 rather than zero, yielding $\epsilon_{\infty} \simeq 32.09$. The 1,A $F_{D}$ distribution-of-dielectricrelaxation- times curve shown in Fig. 3 was obtained by numerically inverting this data set, expressed at the complex dielectric constant level, using Eqs. (1) and (12). For comparison, the 2, A data for inversion involved the parameters obtained from a fit of the 1,A data using the EDRT model. The close agreement between the 1,A and 2,A curves again shows that the EDRT is a good approximation to the distribution of "dielectric" relaxation times associated directly with the original KWW1 data. Because of inaccuracies in the largest- $\tau$ points of the two curves, it is difficult to isolate any significant difference between them, although an accurate EDRT should yield a straight line until the appearance of an abrupt cutoff near $\tau / \tau_{0}=$ 1. $[17,30]$. These inversions included the free parameters $\epsilon_{\infty}$ and $\sigma_{0}$ as part of the full fitting circuit and led to estimated values of them in virtually perfect agreement with those expected. No adequate inversions could be obtained without the inclusion of these parameters since they contributed appreciably to the full data which were inverted. The errors in all the smallest- $\tau$ points for the various curves are clearly evident in this figure.

The points of the $G_{1}$ DRT curve marked $\rho$ 1,A;CSD in Fig. 3 have been fitted directly to the KWW0 distribution using LEVM. Such fitting yields estimates of $\beta_{1}$ and $\tau_{01}$. The $S_{F}$ value using all the points was 0.06 , but with end points eliminated it dropped to 0.0014 and yielded parameter estimates correct to three significant figures or better, well validating the inversion procedure. The curve marked 2,A; CSD involved resistive DRT estimation found by CSD1 inversion of the 2,A EDRT data and is thus an estimate of the Voigt-model distribution following from data generated with the 2, A Maxwell model, one which here involves a distribution of dielectric relaxation times. 


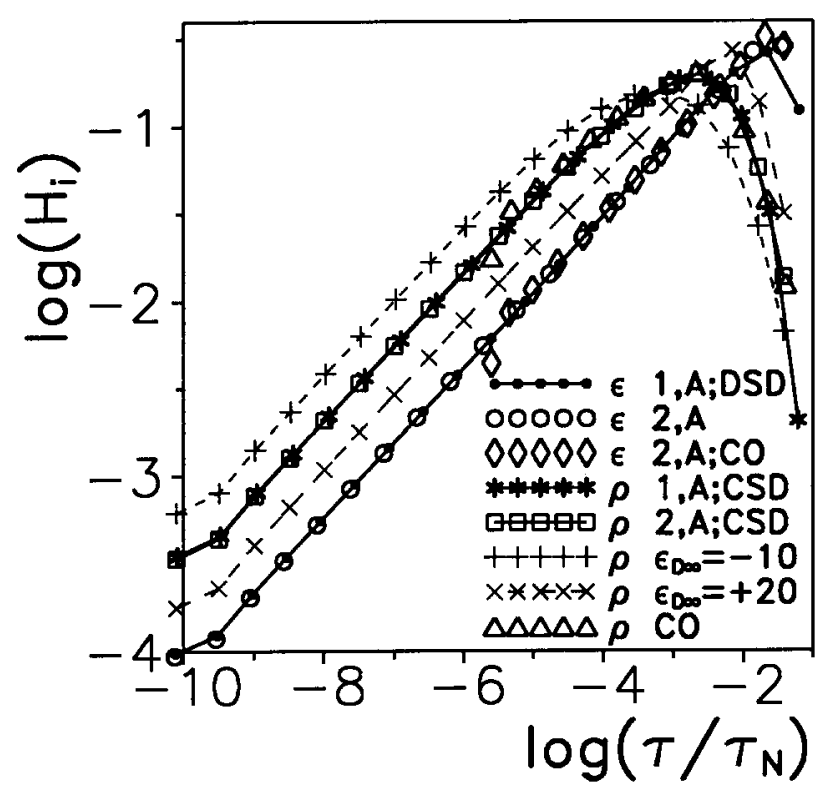

Figure 3. Log-log plots of H; DRT strengths vs. $\tau$. In the legend, $\epsilon$ indicates dielectric-level inversion to estimate dielectric DRTs, while $\rho$ indicates resistivity-level inversion to estimate the related distributions of resistivity relaxation times. Here 1,A and 2,A denote data associated with the A-row of Tables 1 and 2, and CO identifies a cutoff distribution with $y_{\min }=-10 . \epsilon_{D \infty}$ was 10 , except where indicated otherwise.

It is important to note that the 1,A;CSD and 2,A;CSD results represent basic distributions which do not include the effect of any non-zero $\epsilon_{D \infty}$. One obtains exactly the same results inverting data with $\epsilon_{D \infty}=0$ or data with $\epsilon_{D \infty} \neq 0$, provided that the effect of non-zero $\epsilon_{D \infty}$ is properly included in the inversion procedure. Unfortunately, unlike the inversion of data to obtain a distribution of dielectric relaxation times, as discussed above, inversion to obtain a distribution of resistive relaxation times does not allow a quantity such as $\epsilon_{D \infty}$ to be a free parameter, thus it cannot be estimated from the inversion fit but must first be estimated as in the fits of $1, \mathrm{~B}$ or $1, \mathrm{C}$ and then taken as a fixed parameter in the CSD inversion.

The curves in Fig. 3 with $\epsilon_{D \infty}=-10$ and +20 show the effect of not accounting for a non-zero $\epsilon_{D \infty}$ in the inversion. As demonstrated, when $\epsilon_{\infty}<(>) \epsilon_{C \infty}$ the apparent DRT is wider (narrower) than the proper KWW0 distribution and is not well fitted by such a distribution. When $\epsilon_{D \infty}$ is of the order of 100 and is not accounted for in the inversion, the resulting distribution shows a straight-line portion very close to that of the 2,A EDRT one.

Direct fitting to the KWW0 distribution model of the 2,A;CSD results with end points omitted led to
$S_{F} \simeq 0.02$, with parameter estimates equal to those of the original KWW1 model to nearly three significant figures. Thus, although the KWW DRT is not quite the exact CSD distribution related to the DSD EDRT, comparison of the relevant curves in the figure shows that any difference is virtually imperceptible in a log-log plot and suggests that for usual experimental data containing only random errors it would be difficult to verify any difference. Incidentally, although CSD0type inversion of the 2,A EDRT data was carried out, it did not lead to a DRT close to that of the KWW0 but instead to one nearer in form to the original EDRT of curve 2 , A.

Meixner [31], and later Gross [5,8,9], stated that if one of a continuous-distribution DRT pair was truncated within a limited interval, then the other distribution of the pair would be continuous in the same interval and be zero outside, except for the appearance of a single discrete line. Later, Gross [9] presciently pointed out that such lines need not always appear, but he did not explicitly specify the general conditions required for their appearance or absence. Here, we do so below. Incidentally, Gross [9] made the cogent comments that (a) either, but not both, of related Maxwell and Wagner-Voigt models can have physical significance for a given real material, and (b) the discrete lines which sometimes appear have hardly more than mathematical significance. An important purpose of the present work is to find clues from fitting and inversion which may allow one to decide which one of the DRTs in (a) is associated with a physically significant model.

The two curves marked "CO" in Fig. 3 are ones in which the EDRT used in generating the data was cut off by using $y_{\min }=-10$. Now since $\log \left(\tau_{\min } / \tau_{N}\right)=$ $\log \left\{\tau_{0 D} \exp \left(y_{\min }\right) / \tau_{N}\right\}$ with $\tau_{N}=1 \mathrm{~s}$, the result is about -5.68 . The points with smallest $\tau$ on the two $\mathrm{CO}$ curves are both at about -5.60 . As the $M$ value used in the inversion increases, the smallest- $\tau$ value found decreases toward the cutoff limit. The two DRTs are clearly non-zero over the same interval, as required by the earlier work, but no discrete line is found for the distribution of resistive relaxation times, contrary to the earlier predictions. Incidentally, the $S_{F}$ values of LEVM inversion fits of the present type decrease substantially for exact data as $i \rightarrow i+1$ and as the width of the non-zero interval of a continuous distribution decreases. For example, typical inversions such as the 2,A; CSD one of Fig. 3 , where $M=19$, have $S_{F}$ 
values of about $3 \times 10^{-4}$, while the $\mathrm{CO}$ curves with $M=13$ lead to $S_{F}$ values less than $10^{-5}$. These results show how closely the inversion-fit models fit the original frequency-response data and make it clear that with such good fits there is no possibility of missing a discrete line.

\section{DRT inversion estimates: Fig. 4 re- sults}

We have seen that while changes in $\epsilon_{D \infty}$ change the shape of the DRT obtained by inversion of the full data when the presence of $\epsilon_{D \infty}$ is unaccounted for, no discrete line occurs. But since changes in $\epsilon_{D \infty}$ do not directly involve the CSD constitutive equations, Eqs. (58 ), it is worthwhile investigating the effects of changes which do so. Some inversion results, with all non-zero $\epsilon_{D \infty}$ effects properly accounted for, are presented in Fig. 4 for the three different values of $\sigma_{0}$ used in rows A, D, and G of Table 2. Define $r$ as the ratio of the fixed $\sigma_{0}$ values of rows D or G of column 7 of Table 2 to the fixed $\sigma_{0}=\sigma_{C 0}$ value listed in row A. The $r=10$ and $r=0.1, M=19 \mathrm{~A}$ curves are inversion results obtained with no $\sigma_{C P}$ parameter included in the inversion; thus they should not be expected to yield proper DRT estimates.

The two $r=10$ curves demonstrate that changing $M$ does not significantly change the optimized $\tau$ value of the peak point, thus suggesting that this point arises from a discrete line while the other points are associated with a continuous distribution. Similarly, appreciable changes in $M$ for the first $r=0.1$ curve do not change the position of the circular-symbol point enclosed in the square, thus verifying that this point also represents a single discrete DRT.

Suppose that we now account for the need for a nonzero $\sigma_{C P}$ by including such a separate, parallel conductivity parameter in the full inversion model. For the $r=10$ results, one finds, as expected, that the inclusion of such a parameter with a fixed value of $69.02 \times 10^{-10}$ $(\Omega-\mathrm{cm})^{-1}$ results in a DRT estimate in full agreement with that designated by 2,A; CSD in Fig. 3. Here that curve is reproduced as the solid line labeled $r=1$, $M=19$ and is shown without any points included. Thus, no discrete line appears when the effect of $\sigma_{C P}$ is properly taken into account.

But what happens for the $r=0.1$ situation when a negative $\sigma_{C P}$ is included? Again one finds that the inversion points approach the usual CSD $r=1$,
$M=19$ distribution as $\sigma_{c p}$ decreases from zero toward the Table- 2 row-H fit value of $-6.046 \times 10^{-10}(\Omega-\mathrm{cm})^{-1}$. Inversion with a non-zero value of $\sigma_{c p}$ near that above is difficult because it involves the small difference between two nearly equal large numbers. The points identified by $r=0.1, M=19 B$ in the figure are the largest eight $\tau$ values found in the inversion when the parallel conductivity used in the inversion was set to the intermediate value of $-1.7 \times 10^{-10}(\Omega-\mathrm{cm})^{-1}$. The other points of the 19 fell very closely on the $r=1$ line. Here the last point on the right is still a discrete-distribution one, but we see that, as the added parallel conductivity becomes more negative, all the higher- $\tau$ points approach the $r=1$ line as the proper value of $\sigma_{C P}$ is approached. For this value, the discrete point has disappeared and all points are those of the expected continuous distribution. The present results suggest that the presence of a discrete line in previous analytical calculations of a CSD DRT arose because the need for $\sigma_{C P}$ was unrecognized and thus was not included in the analysis.

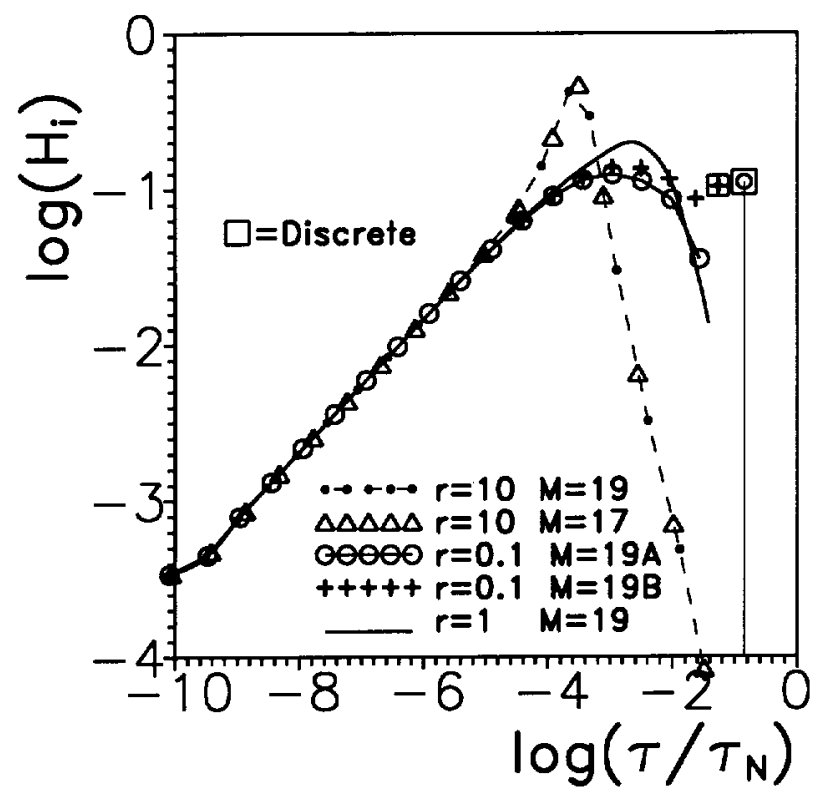

Figure 4. Inversion results similar to those of Fig. 3 except that the effects are demonstrated of three different choices for the conductivity ratio $r$ defined in the Fig.-2 caption and used in generating the data. All inversion results shown were carried out with the parallel conductivity, $\sigma_{C P}$ taken zero except for the $M=19 B$ curve, where $\sigma_{C P}$ was fixed at $-1.7 \times 10^{-10}(\Omega-\mathrm{cm})^{-1}$. With the proper $\sigma_{C P}$ values used in the inversions (see Table 2), the $r=0.1$ and $r=10$ results agreed with the KWW0-DRT $r=1$ curve shown. All points shown here are associated with continuous distributions except those enclosed in squares. 


\section{DRT inversion estimates: Fig. 5 re- sults}

Kita [28] was unable to obtain analytically a DCRT expression from a particular distribution of dielectric relaxation times, that of Davidson and Cole [DC] [32], which satisfied the principle of equivalence of Maxwell and Wagner circuits. See the Appendix for details about this important principle. Gross $[8,9]$ showed that the principle was valid if the calculated Wagner-Voigt DRT included a discrete line, shown to arise from the intrinsic cutoff (truncation) of the DC distribution at $\tau>\tau_{0 D}$. Incidentally, Kita's work also suggests that the CSD response most appropriately associated with a Maxwell-model DSD data set is of CSD1 rather than CSD0 type.

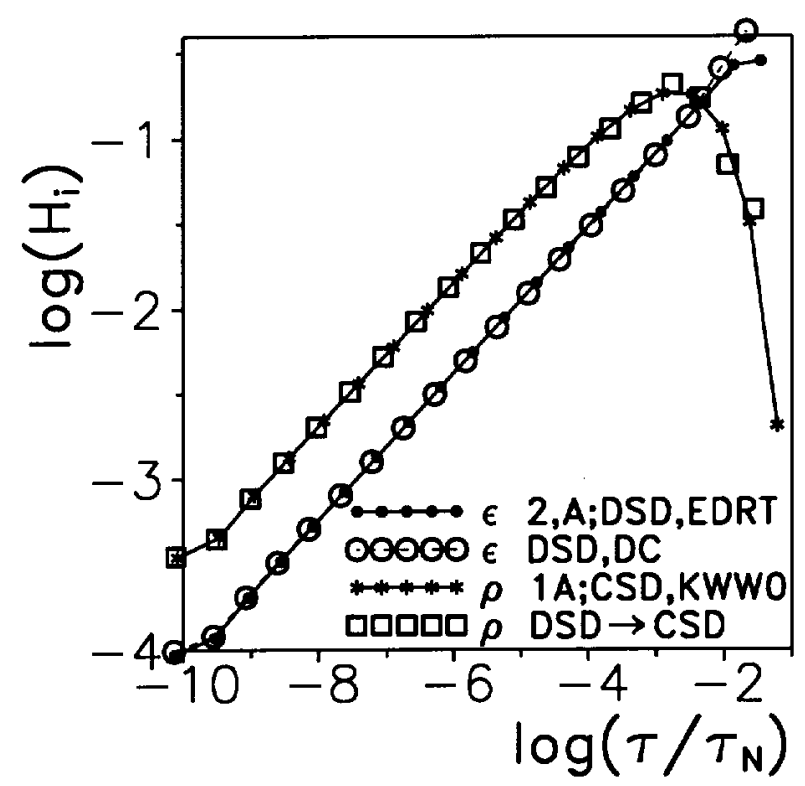

Figure 5. Inversion results for the dielectric DRTs (designated with $\epsilon$ ): (a) the 2,A exponential DRT, EDRT, repeated from Fig.3, and (b) the Davidson-Cole DRT, DC. Also shown are CSD-inversion DRT estimates associated with (b), marked DSD $\rightarrow$ CSD, and the 1,A,CSD KWW0 DRT, also repeated from Fig.3, both designated with $\rho$.

Since we have demonstrated earlier that abrupt cutoff of an exponential distribution at a small $\tau_{\min }$ value does not lead to the appearance of a discrete line, it is worthwhile to apply the present methods to estimate points of a DDRT, starting with DC data at the $\epsilon$ level. First, however, for the convenience of the reader in comparing the work of Gross and Kita, it is worth pointing out that Kita's symbol $\epsilon$ denotes the dielectric permittivity, here $\epsilon \epsilon_{V}$, while Gross [8] defines $\epsilon^{*}$ as the complex dielectric constant but then omits a necessary $\epsilon_{V}$ term in his admittance and time-constant expressions.
For easy comparison, two DRT curves previously presented and discussed are included in Fig. 5. The curve marked DSD,DC is the distribution of dielectric relaxation times estimated by inversion of exact DC data. The original KWW1 frequency-response data were fitted using the DC model (with a somewhat less accurate fit found than that obtained with the EDRT model), and then exact DSD data were generated with the DC model using the values of the appropriate parameters of Section III-A, except that $\tau_{0 D}$ was set to $0.025 \mathrm{~s}$, the approximate fit value. Comparison of the first two curves identified in the figure indicates extremely good agreement between the EDRT DRT estimate and that for the DC situation except for the three highest- $\tau$ points. As expected, for the DC curve we see the approach to an infinite value at cutoff, but because of the problem with end-point estimates, the last two points do not rise as fast as required for an exact DC DRT. The maximum- $\tau$ estimate with $M=19$ was about $0.021 \mathrm{~s}$, and it was found to approach closer and closer to the cutoff value of $0.025 \mathrm{~s}$ as the value of $M$ used in the inversion was increased.

The last two curves in Fig. 5 compare the practically exact KWW0 $F_{0}$, or, equivalently, the KWW1 $G_{1}$ distribution, with the CSD1 inversion estimate of $G_{1}$ associated with the exact DSD DC data. We see that although the agreement is not quite as close as that for the comparable EDRT $\rho$-level comparison of Fig. 3 , the agreement is still sufficiently good that for typical experimental data one would not be able to distinguish well between the three different DRT estimates. Although fitting comparisons for DDRT estimates obtained from the same data might allow somewhat better discrimination, the most appropriate fitting model can be much better identified from direct fitting in the frequency domain of original frequency-response data. The present results again confirm that accurate inversion estimates of truncated DSD DRTs need not lead to the appearance of a discrete response line.

\section{Summary and conclusions}

It has been shown that frequency-response data associated with a model of a leaky, dispersive dielectric material can be fitted with very high accuracy by a conductive-system response expression. Therefore, one cannot use CSD and DSD fitting of a given data set to determine unambiguously whether observed electrical dispersion is associated with relaxing dipoles or with 
mobile charges. This conclusion is foreshadowed by earlier work [3] in which it was demonstrated that the limiting high- and low-frequency log-log slopes of all immittance responses are exactly the same for DSD and CSD1 response when $\beta_{1}=\beta_{D}$ and for DSD and CSD0 response when $\beta_{0}=1-\beta_{D}$, provided the DSD response involved $\sigma_{0} \neq 0$ and the CSD response involved $\epsilon_{D \infty} \neq 0$.

Here, we find that the requirement that CSD and DSD approaches (possibly involving conductive or dielectric distributions of relaxation times, respectively) be able to fit data equally well necessitates an augmentation of the Wagner-Voigt relaxation circuit usually considered in applying the principle of equivalence of Maxwell and Wagner-Voigt circuits. CSD model response, Eq. (11), involving a discrete or continuous distribution of conductive relaxation times in the WagnerVoigt circuit, must not only be augmented by the effects of a parallel specific susceptance (associated with the limiting dielectric constant $\left.\epsilon_{D \infty}\right)[2,3,23]$, but also by a parallel conductivity, here designated as $\sigma_{C P}$. See the results presented in Table 2. In exceptional cases, $\sigma_{C P}$, which is not a separate parameter in DSD fitting, must be negative to allow adequate CSD fitting of arbitrary DSD data. When a $\sigma_{C P}$ estimate is negative, it is highly unlikely that the response of the physical system investigated is dominated by dispersion associated with mobile charges, rather than by purely dielectric effects, and it is still unlikely even when $\sigma_{C P}$ is positive and significant compared to $\sigma_{0}$. Thus, though discrimination is not unambiguously possible when $\sigma_{C P} \neq 0$, it is nevertheless plausible. Conversely, when an estimate of $\sigma_{C P}$ is statistically indistinguishable from zero, the data may, in principle, be equally well fitted by a CSD or a DSD approach, thus precluding discrimination using only a single data set.

The present analysis leads to the important conclusion that if CSD data involves KWW1 $I_{1}(\omega)$ dispersive response, then the EDRT $I_{D}(\omega)$ response model is an appropriate choice for DSD fitting of the data using
Eq. (12). Although the use of the Davidson-Cole $I_{D}(\omega)$ model for generating DSD data also leads rather closely to KWW1 CSD response, somewhat better results are found for the KWW1, EDRT pair.

The prediction $[8,9,31]$ that truncating a full-range DSD DRT, or using the DC, which involves an intrinsically truncated distribution, leads to CSD response with both a continuous DRT and a discrete response line has not been verified by the present work. It has, however, been found that when $\sigma_{C P}$ is not accounted for in the inversion, such a line appears whether the original DSD DDRT is truncated or not. When inversion of frequency-reponse data is used to estimate a CSD DCRT, taking proper account in the fitting procedure of non-zero $\epsilon_{D \infty}$ and $\sigma_{C P}$ parameters allows one to obtain an accurate estimate of the true continuous DRT involved, but omission of such parameters in the inversion leads to erroneous estimates, as shown in Figs. 3 and 4. None of the earlier analytical DRT work $[8,9,28,31]$ took explicit account of these parameters, possibly explaining the present discrepancies.

Although DSD and CSD inversion results are instructive and can be useful for distinguishing between continuous and discrete parts of a DRT, it appears that direct fitting of frequency- (or temporal- $[2,25]$ ) response data is likely to allow better discrimination between various possible dispersive models for DSD or CSD fitting, and, in some cases, it can lead to a plausible conclusion about the identity of the dominant physical processes involved in the dispersion. Measurements over a range of temperatures should allow even better decisions about the character of the dominant dispersion process to be reached.

\section{Acknowledgment}

I am happy to acknowledge the pleasure and understanding I have gained from reading the linear-system papers of Professor Bernhard Gross since I started work in the field in 1949 . 


\section{Definition of Acronyms}

$\begin{array}{ll}\text { ac } & \text { Alternating current } \\ \text { CSD } & \text { Conductive-system dispersion } \\ \text { CSDn } & \mathrm{n}=0 \text { or } 1 \text {; see below for definitions of the n values } \\ \mathrm{dc} & \text { Direct current } \\ \mathrm{DC} & \text { Davidson-Cole response model } \\ \text { DCRT } & \text { Distribution of conductive-system relaxation times } \\ \text { DDRT } & \text { Distribution of dielectric-system relaxation times } \\ \text { DRT } & \text { Distribution of relaxation times } \\ \text { DSD } & \text { Dielectric-system dispersion } \\ \text { EDRT } & \text { Exponential distribution of relaxation times } \\ \text { KWW } & \text { Kohlrausch-Williams-Watts response model } \\ \text { KWWn } & \text { KWW response defined by index n, where } \mathrm{n}=\text { D, 0, or 1 (see below) } \\ \text { LEVM } & \text { The complex-nonlinear-least-squares fitting program used herein } \\ \mathrm{n}=\mathrm{D} & \text { The response is of DSD character involving a DDRT } \\ \mathrm{n}=\mathrm{C} & \text { The response is of CSD character involving a DCRT and } \mathrm{n}=0 \text { or } 1 \\ \mathrm{n}=0 & \text { The response is of CSDO character, possibly involving a DCRT formally } \\ & \text { equivalent to a given DDRT } \\ \mathrm{n}=1 & \text { The response is of CSDl character involving a DCRT related to a given } \\ & \text { CSDO DCRT by Eq. (13) of the text }\end{array}$

\section{APPENDIX}

Because of the importance of the principle of equivalence for relaxation systems, it is worthwhile to summarize some historical information about it, most of which appears in Ref. 33. Two two-terminal passive circuits with time-invariant elements are said to be equivalent when their responses over the full frequency range are exactly the same. Consider pure reactance circuits first, ones made up only of non-dissipative ideal capacitative and inductive elements. In 1924 Foster [34] proved that any such reactance system can be equivalently represented by a parallel combination of resonant elements (capacitance and inductance in series) or by a series combination of antiresonant elements (capacitance and inductance in parallel). To account properly for pole and zero behavior at zero and infinite frequencies, single elements must also be included in order to obtain full equivalence [35].

Later, Bode [36] showed that the Foster reactance theorem could be generalized by a frequencytransformation method. For a relaxation situation, his procedure replaces inductances in the original reactance system by resistances. This procedure can thus lead to Maxwell and Wagner-Voigt circuits which are equivalent. As shown herein, for full equivalence of dielectric- system response to conductive-system response (in which the effective resistance at infinite frequency associated with the dispersion process is zero), the Maxwell circuit must, in general, include a capacitor and a resistor in parallel with the rest of the circuit, and the Wagner-Voigt circuit must also include such parallel elements. The parallel capacitance represents the high- frequency-limiting dielectric constant of the system, and the parallel resistances are different for the two cases. Actual transformations from one circuit to the other are best carried out by complex-nonlinearleast-squares fitting [27], but Novoseleskii et. al. [37] have provided some useful analytical expressions for doing so for circuits with only three or four elements. The present work demonstrates that the principle of equivalence applies for continuous distributions as well.

\section{References}

1. J. R. Macdonald, J. Appl. Phys. 58, 1955 (1985).

2. J. R. Macdonald, J. Non-Cryst. Solids 197, 83 (1996); erratum: ibid 204, 309 (1996), and $G_{D}$ in Eq. (A2) should be $G_{C D}$, the present $G_{1}$ quantity.

3. J. R. Macdonald, J. Appl. Phys. 82, 3962 (1997).

4. J. R. Macdonald, in Electrically Based Microstructural Characterization, Proceedings, p. 71, Vol. 411, Fall meeting, November 1995, Boston, MA (Materials Research Society, Pittsburgh, PA, 1996).

5. B. Gross, J. Appl. Phys. 18, 212 (1947); ibid 19, 257 (1948), ibid 62, 2763 (1987).

6. B. Gross and H. Pelzer, J. Appl. Phys. 22, 1035 (1951).

7. B. Gross, Mathematical Structure of the Theories of Viscoelasticity (Hermann \& Cie, Paris, France, 1953).

8. B. Gross, J. Appl. Phys. 57, 2331 (1985).

9. B. Gross, J. Appl. Phys. 67, 6399 (1990). 
10. J. R. Macdonald and C. A. Barlow, Jr., Rev. Mod. Phys. 35, 940 (1963).

11. J. R. Macdonald, J. Chem. Phys. 102, 6241 (1995); Computers in Physics 9, 546 (1995).

12. A. R. Long, Adv. Phys. 31, 553 (1982).

13. J. R. Macdonald, J. Appl. Phys. 62, R51 (1987).

14. S. R. Elliott, Adv. in Phys. 36, 135 (1987).

15. S. R. Elliott, Solid State Ionics 70\& 71, 27 (1994).

16. J. R. Macdonald, Phys. Rev. B 49, 9428 (1994II).

17. J. R. Macdonald and J. C. Wang, Solid State Ionics 60, 319 (1993).

18. J. R. Macdonald, J. Non-Cryst. Solids 210, 70 (1997).

19. S. R. Elliott, Phil. Mag. 36, 1291 (1977).

20. A. K. Jonscher, Dielectric Relaxation in Solids (Chelsea Dielectric, London, 1983).

21. L. A. Dissado and R. M. Hill, J. Chem. Soc. Faraday Trans. 2 80, 291 (1984).

22. J. C. Giuntini, J. V. Zanchetta, and F. Henn, Solid State Ionics 28-30, 142 (1988).

23. J. R. Macdonald, J. Non-Cryst. Solids 212, 95 (1997). The symbol $\sigma_{0}$ should be removed from the right end of Eq. (12); Eq. (7) in this work does not apply to Eqs. (3) and (4) when $\sigma_{C \infty} \neq 0$ (see Ref. 2, above); and the word "frequency" in the third line from the bottom of the seconf column of p.111 should be "temperature".

24. J. R. Macdonald, J. Chem. Phys. 36, 345 (1962).

25. J. R. Macdonald, J. Appl. Phys. 84, 812 (1998). The word "out" in the third line from the bottom of the first column on p.820 should be "but".

26. R. Kohlrausch, Pogg. Ann. der Phys. und Chemie, (2) 91, 179 (1854); G. Williams and D.
C. Watts, Trans. Faraday Soc. 66, 80 (1970), G. Williams, D. C. Watts, S. B. Dev, and A. M. North, Trans. Faraday Soc. 67, 1323 (1971).

27. J. R. Macdonald and L. D. Potter, Jr., Solid State Ionics 23, 61 (1987). The latest version of the LEVM fitting program, V. 7.1, may be obtained at no cost from Solartron Instruments, Victoria Road, Farnborough, Hampshire, GU147PW, England; e-mail, attention Mary Cutler, lab-info@solartron.com. More details about the program appear in http://www.physics.unc.edu/ macd/.

28. Y. Kita, J. Appl. Phys. 55, 3747 (1984).

29. E. W. Montroll and J. T. Bendler, J. Stat. Physics 14, 129 (1984).

30. B. A. Boukamp and J. R. Macdonald, Solid State Ionics 74, 85 (1994).

31. J. Meixner, Kolloid Z. 134, 3 (1953).

32. D. W. Davidson and R. H. Cole, J. Chem. Phys. 19, 1484 (1951).

33. B. Gross and M. T. de Figueiredo, J. Appl. Phys. D 18, 617 (1985).

34. R. M. Foster, Bell Syst. Tech. J. April, 259 (1924).

35. H. W. Bode, Network Analysis and Feedback Amplifier Design (New York, Van Nostrand, 1945), pp. 181-182.

36. H. W. Bode, Network Analysis and Feedback Amplifier Design (New York, Van Nostrand, 1945), pp. 214-215.

37. I. M. Novoseleskii, N. N. Gudina and Yu. I. Fetistov, Sov. Electrochem. 8, 546 (1972). 